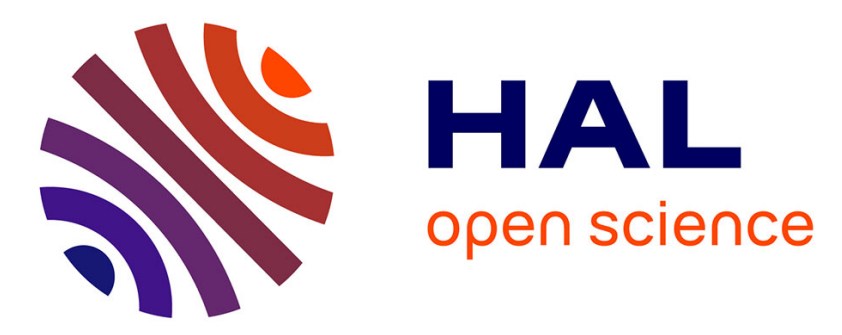

\title{
Tuning photoinduced terahertz conductivity in monolayer graphene: Optical-pump terahertz-probe spectroscopy
}

\author{
Srabani Kar, Dipti R. Mohapatra, Eric Freysz, Ajay Kumar Sood
}

\section{To cite this version:}

Srabani Kar, Dipti R. Mohapatra, Eric Freysz, Ajay Kumar Sood. Tuning photoinduced terahertz conductivity in monolayer graphene: Optical-pump terahertz-probe spectroscopy. Physical Review B: Condensed Matter and Materials Physics (1998-2015), 2014, 90 (16), pp.165420 (1-9). 10.1103/PhysRevB.90.165420 . hal-01094441

\section{HAL Id: hal-01094441 \\ https://hal.science/hal-01094441}

Submitted on 12 Dec 2014

HAL is a multi-disciplinary open access archive for the deposit and dissemination of scientific research documents, whether they are published or not. The documents may come from teaching and research institutions in France or abroad, or from public or private research centers.
L'archive ouverte pluridisciplinaire HAL, est destinée au dépôt et à la diffusion de documents scientifiques de niveau recherche, publiés ou non, émanant des établissements d'enseignement et de recherche français ou étrangers, des laboratoires publics ou privés.

\section{(ㄷ)(1) $\$$}

Distributed under a Creative Commons Attribution - NonCommerciall 4.0 International 


\title{
Tuning photoinduced terahertz conductivity in monolayer graphene: Optical-pump terahertz-probe spectroscopy
}

\author{
Srabani Kar, ${ }^{1,2}$ Dipti R. Mohapatra, ${ }^{1}$ Eric Freysz, ${ }^{3}$ and A. K. Sood ${ }^{1,2, *}$ \\ ${ }^{1}$ Department of Physics, Indian Institute of Science, Bangalore 560 012, India \\ ${ }^{2}$ Center for Ultrafast Laser Applications, Indian Institute of Science, Bangalore 560 012, India \\ ${ }^{3}$ University of Bordeaux, LOMA, UMR CNRS 5798, 351, Cours de la liberation, 33405 Talence cedex, France
}

(Received 11 June 2014; revised manuscript received 16 August 2014; published 16 October 2014)

\begin{abstract}
Optical-pump terahertz-probe differential transmission measurements of as-prepared single layer graphene (AG) (unintentionally hole doped with Fermi energy $E_{F}$ at $\sim-180 \mathrm{meV}$ ), nitrogen doping compensated graphene (NDG) with $E_{F} \sim-10 \mathrm{meV}$, and thermally annealed doped graphene (TAG) are examined quantitatively to understand the opposite signs of photoinduced dynamic terahertz conductivity $\Delta \sigma$. It is negative for AG and TAG but positive for NDG. We show that the recently proposed mechanism of multiple generations of secondary hot carriers due to Coulomb interaction of photoexcited carriers with the existing carriers together with the intraband scattering can explain the change of photoinduced conductivity sign and its magnitude. We give a quantitative estimate of $\Delta \sigma$ in terms of controlling parameters-the Fermi energy $E_{F}$ and momentum relaxation time $\tau$. Furthermore, the cooling of photoexcited carriers is analyzed using a supercollision model which involves a defect mediated collision of the hot carriers with the acoustic phonons, thus giving an estimate of the deformation potential.
\end{abstract}

DOI: 10.1103/PhysRevB.90.165420

\section{INTRODUCTION}

The performance of electronic and optoelectronic devices depends on the material properties such as carrier mobility, energy conversion efficiency from photon to electron-hole pairs, spectral response, and equilibration of the photogenerated carriers. The linear band dispersion with zero band gap in monolayer graphene responsible for many fascinating transport phenomena and optical effects makes graphene a desirable material for high speed optoelectronic devices [1-7]. A key question to answer in optoelectronic applications is the relaxation of the hot carriers in conical energy-momentum space of the monolayer graphene. Ultrafast time resolved pump-probe spectroscopy [8-12] and angle resolved photoemission spectroscopy [13] have been shown to be excellent probes of nonequilibrium carrier dynamics in graphene. The terahertz-probe pulse following the optical pump examines the intraband scattering dynamics as compared to optical probe which is sensitive to both interband and intraband scattering processes. Following the pump pulse, the photoexcited carriers achieve a quasiequilibrium Fermi-Dirac distribution, characterized by electron temperature $T_{e}$, mostly by carrier-carrier scattering. The cooling of the carriers can occur through phonon emission as well as through carrier-carrier scattering. The latter involves the transfer of energy of photoexcited carriers to the existing carriers in graphene (making them hot) [14-16]. The cooling via phonon emission involving optical phonons occurs on a time scale of $\sim 100$ to $500 \mathrm{fs}$ till the energy of the photoexcited carriers is less than the optical phonon energy $(\sim 200 \mathrm{meV})$. This is followed by the direct coupling between the carriers and acoustic phonons which can last for tens of picoseconds. However, the carrieracoustic phonon relaxation time is reduced to less than $10 \mathrm{ps}$ when large momentum and large energy acoustic phonons

\footnotetext{
*Corresponding author: asood@physics.iisc.ernet.in
}

PACS number(s): 81.05.ue, 78.47.-p, 78.20.Ci, 78.67.Wj

emission occurs mediated by the disorder. This three body (carriers + acoustic phonons + disorder) mediated cooling is termed as supercollision cooling of the carriers [17]. Several groups have reported optical-pump terahertz-probe (OPTP) time domain spectroscopy of epitaxial grown as well as CVD grown graphene showing positive $[9,10,18,19]$ as well as negative dynamic conductivity [16,20-22]. The positive dynamic conductivity can be easily understood in terms of intraband scattering of the carriers. Docherty et al. [20] showed that the $\mathrm{THz}$ photoconductivity changes from positive in vacuum to negative in nitrogen, air, and oxygen environment and proposed stimulated $\mathrm{THz}$ emission from photoexcited graphene as the cause of the negative photoconductivity. Several other experimental [23] and theoretical [24,25] studies have also attributed the negative dynamic conductivity to the amplified stimulated terahertz emission above a threshold pump intensity [26]. In this context, Gierz et al. [13] have shown experimentally that only within 130 fs after photoexcitation, the Fermi-Dirac distribution for electron and holes are different, suggesting that population inversion and hence stimulated emission is not feasible beyond this time window. Jnawali et al. [21] have attributed the decrease in photoconductivity to the increase in carrier scattering rate with negligible increase of Drude weight. Tielrooij et al. [16] have proposed to explain the negative dynamic conductivity via Coulomb interaction governed carrier-carrier scattering where the energy of the photoexcited carriers is transferred to the existing carriers in the Dirac cone, a process termed as secondary hot carrier generation (SHCG). In a very recent study of tuning the sign of OPTP signal from the single layer graphene (SLG) using electrostatic top gating, [27] the explanation given is as follows: Taking conductivity $\sigma=\mathfrak{D} / \Gamma$, the dynamic conductivity $\Delta \sigma=\left(\Delta \mathfrak{D} / \mathfrak{D}_{0}\right) \sigma_{0}-\left(\Delta \Gamma / \Gamma_{0}\right) \sigma_{0}$, where $\mathfrak{D}$ is the Drude weight, $\Gamma$ is the carrier scattering rate, and the subscript 0 stands for the pump off condition. The contribution from the Drude weight dominates near the charge neutral point and is positive. For higher doping, the contribution from 
change of scattering rate $\Delta \sigma_{\Gamma}=-\left(\Delta \Gamma / \Gamma_{0}\right) \sigma_{0}$ controls the $\Delta \sigma$. The authors [27] assumed $\Delta \Gamma / \Gamma_{0}=0.2$, independent of $E_{F}$, to explain the negative dynamic conductivity. This framework does not explain the observed saturation behavior of the dynamic conductivity at higher Fermi energy. Therefore, the issue of the optical-pump induced terahertz conductivity, in particular its sign and amplitude, is still open and needs to be understood quantitatively.

In this work we report the $\mathrm{THz}$ conductivity $\sigma(\omega)$ of (1) as-grown single layer graphene (AG) by chemical vapor deposition which is unintentionally hole doped, (2) nitrogen doping compensated monolayer graphene (NDG), and (3) thermally annealed nitrogen doped graphene (TAG). The samples have different values of carrier momentum relaxation time $\tau$ and the Fermi energy $E_{F}$. The conductivities obtained from the $\mathrm{THz}$ transmission measurements compare well with the estimates from the relative Raman intensities of $D$ and $G$ bands. Next, we present optical pump $(1.58 \mathrm{eV}) \mathrm{THz}$ probe measurements of the transient $\mathrm{THz}$ photoconductive response of the graphene samples. On photoexcitation, the dynamic conductivity $[\Delta \sigma=\sigma$ (pump on $)-\sigma$ (pump off)] is negative for the AG but positive for the NDG. In both cases, the $|\Delta \sigma|^{\max }$ is $\sim 1.5 G_{0}$, where $G_{0}=2 e^{2} / h(=77.3 \mu S)$ is quantum of conductance. We show that on thermal annealing the NDG, the $\Delta \sigma$ is once again negative. A quantitative analysis of $\Delta \sigma$ is done by noting that in the terahertz range, intraband scattering contribution to $\Delta \sigma$ is orders of magnitude larger than the interband contribution. We invoke secondary hot carrier generation (SHCG) [16] to explain quantitatively the negative $\Delta \sigma$ in AG. The sign and magnitude of the dynamic conductivity in graphene thus depends on the relative contributions of the intraband scattering and the SHCG, which, in turn, depend on the momentum relaxation time and the Fermi energy. The cooling of hot carriers is quantitatively analyzed in terms of supercollision (SC) processes. The frequency dependence of dynamic conductivity is also measured and analyzed in terms of the Drude-Lorentz model.

\section{METHOD}

\section{A. Terahertz setup}

The output beam of the Ti:sapphire regenerative amplifier laser system which produces $\sim 50$ fs optical pulses at a central wavelength of $785 \mathrm{~nm}$ with a repetition rate of $1 \mathrm{kHz}$ is divided into three parts: for terahertz generation, terahertz detection, and optical pumping. The terahertz radiation is generated by co-focusing the fundamental and its second harmonic beam using a $10 \mathrm{~cm}$ focal length lens to produce plasma in air. The unwanted light following the plasma was blocked using a high resistive silicon wafer. The terahertz was collimated and focused on the sample by a pair of off-axis parabolic mirrors and again collimated and focused by parabolic mirrors on a $1 \mathrm{~mm}$ thick ZnTe crystal used as a detector. All experiments were carried out in transmission geometry in a nitrogen environment at room temperature. The estimated terahertz electric field is $\sim 30 \mathrm{kV} / \mathrm{cm}$. The optical-pump-induced changes in the terahertz transmission were measured at the maximum position of the terahertz electric field. The spot size of the pump beam was $\sim 0.7 \mathrm{~cm}$ and the spot size of the terahertz beam was $\sim 0.3 \mathrm{~cm}$ so that the pump can excite the sample uniformly. To measure the photoinduced transmission the chopper $(341 \mathrm{~Hz})$ was placed in the pump path and to measure the $\mathrm{THz}$ electric field from the unexcited sample, the chopper was placed in the path of the terahertz beam.

\section{B. Sample preparation}

The graphene samples were grown by using the wellknown chemical vapor deposition (CVD) method on $25 \mu \mathrm{m}$ thick copper foil [28]. Before growth, the copper foils were cleaned through a chemical process using acetone, acetic acid, deionized water, isopropyl alcohol, and methanol successively. In order to remove the oxides and chemical residues, the copper substrates were heated at $1000{ }^{\circ} \mathrm{C}$ for $30 \mathrm{~min}$ in the presence of hydrogen at a pressure of 20 Torr. Subsequently, methane was flowed into the chamber and graphene growth was carried out for 30 min keeping hydrogen and methane at a fixed ratio of $1: 3$, followed by stoppage of the methane flow, and the system was allowed to cool at a rate of $20^{\circ} \mathrm{C} / \mathrm{min}$ for first $20 \mathrm{~min}$ without changing the hydrogen flow rate. Then, the system was cooled by a normal fan up to a temperature of $350{ }^{\circ} \mathrm{C}$ in $1 \mathrm{~h}$, followed by natural cooling to room temperature. For nitrogen doping, protocol was similar to that reported recently [29]. Namely, after stopping the methane flow and cooling the system to $850^{\circ} \mathrm{C}$, ammonia $(10 \mathrm{sccm})$ was passed for $10 \mathrm{~min}$. Subsequently, ammonia flow was stopped and the system was cooled in a hydrogen atmosphere as described before. Graphene was transferred onto the $1 \mathrm{~mm}$ thick $\alpha$ quartz by the PMMA technique [30].

\section{RESULTS AND DISCUSSIONS}

\section{A. Characterization of the samples}

Primarily, the experiments were carried out on two samples: AG and NDG. Later, to check the effect of temperature annealing, the NDG was annealed at $400^{\circ} \mathrm{C}$ for $4 \mathrm{~h}$ in argon atmosphere and all the experiments were also performed on this thermally annealed graphene (TAG). The X-ray photoemission spectroscopy (XPS) was done to confirm the presence and nature of nitrogen in graphene. The samples were further characterized by Raman spectroscopy at room temperature using $\lambda=514 \mathrm{~nm}$ of laser light.

The C $1 s$ XPS spectra for AG and NDG are shown in Fig. 1(a). The peak occurs at a binding energy of $284.6 \mathrm{eV}$ for AG corresponding to $s p^{2}$ carbon [31]. For NDG the peaks are at $284.8,285.8$, and $288.5 \mathrm{eV}$ corresponding to $s p^{2}$ carbon, $\mathrm{C}-\mathrm{N}$ and $\mathrm{C}=\mathrm{O}$ bonds [31]. The $\mathrm{N} 1 s$ XPS spectra of NDG sample shown in Fig. 1(b) is fitted with two peaks corresponding to nongraphitic substitution, namely pyridinic $(\sim 399.5 \mathrm{eV})$ and pyrrolic $(\sim 400.5 \mathrm{eV}) \mathrm{N}[31,32]$. In the pyridinic and pyrrolic structures the $\mathrm{N}$ atom bonds with two carbon atoms. We cannot resolve any quaternary (graphitic) substitution of $\mathrm{N}$ (generally occurs at a binding energy of $\sim 401 \mathrm{eV}$ ). Raman spectra of the samples are recorded at room temperature using the excitation wavelength $\lambda=514 \mathrm{~nm}$, displaying four bands [Fig. 1(c)] where peak positions and relative intensities are given in Table I. The $G$ band is a symmetry allowed $E_{2 g}$ zone-center $\Gamma$ point optical phonon and the $D$ band is 
(a)

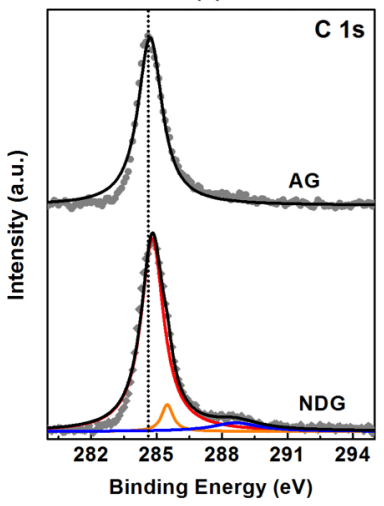

(b)

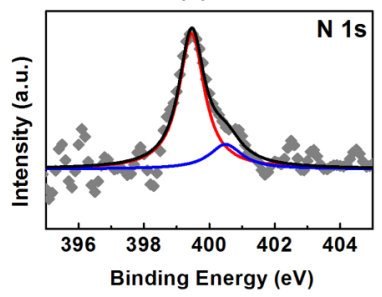

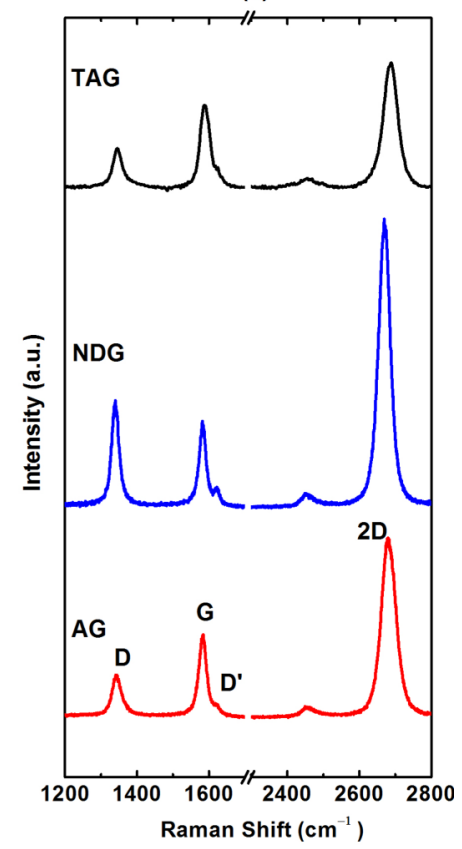

FIG. 1. (Color online) (a) XPS spectra of AG and NDG showing the $\mathrm{C} 1 s$ line. The main peak at $284.6 \mathrm{eV}$ represents $s p^{2} \mathrm{C}$. Two additional peaks appeared at 285.8 and $288.6 \mathrm{eV}$ corresponding to $\mathrm{C}-\mathrm{N}$ and $\mathrm{C}=\mathrm{O}$ bonds. (b) The N 1s XPS spectra of NDG showing two peak at 399.5 and $400.5 \mathrm{eV}$, which suggest the presence of nongraphitic $\mathrm{N}-\mathrm{C}$ bonding. (c) Raman spectra of $\mathrm{AG}, \mathrm{NDG}$, and TAG.

associated with the disorder activated near zone-boundary ( $K$ point) transverse optical phonon [33]. The $2 D$ band is due to second-order Raman scattering from near $K$-point transverse optical phonons and is an unambiguous fingerprint of the number of layers, as understood by double resonance Raman scattering [33]. The $D^{\prime}$ band is associated with the disorder activated near $\Gamma$-point longitudinal optical phonons. The frequencies of the $2 D$ and $G$ bands and their relative intensities depend on the doping levels [34]. It is known that CVD grown graphene can be unintentionally $p$ doped due to charge transfer from $\mathrm{H}_{2} \mathrm{O} / \mathrm{O}_{2}$ molecules [35]. Using the intensity ratio of the $2 D$ and $G$ bands $I(2 D) / I(G)$, the Fermi energy of the AG graphene is $E_{F} \sim-180 \mathrm{meV}$ [34]. After $\mathrm{N}$ doping the intensity ratio $I(2 D) / I(G)$ increases which suggests [34] that the $\mathrm{N}$ doping has compensated the $p$ doping in the AG shifting the Fermi level close to Dirac point with $E_{F} \sim-10 \mathrm{meV}$. However, the exact value of $E_{F}$ is difficult to estimate from the Raman data of NDG. The increase in the intensity of the $D$ and $D^{\prime}$ bands after nitrogen doping clearly reveals an increase of the disorder in NDG. The ratio of the $D$ and $G$ band integrated intensities

$A(D) / A(G)$ increase from $\sim 0.6$ in $\mathrm{AG}$ to $\sim 1.3$ in NDG. The ratio $A(D) / A(G)$ is empirically related [36] to the in-plane crystalline grain size without defects $\left(L_{a}\right)$ as $A(D) / A(G)=$ $\left[2.4 \times 10^{-10} \mathrm{~nm}^{3}\right] \lambda^{4} L_{a}^{-1}$, which gives $L_{a} \sim 34 \mathrm{~nm}$ for the AG, $\sim 15 \mathrm{~nm}$ for the NDG, and $\sim 38 \mathrm{~nm}$ for the TAG. Assuming the transport mean free path of the carriers $\ell \sim L_{a}$ and using Fermi velocity $v_{F} \approx 1 \times 10^{6} \mathrm{~m} / \mathrm{s}$ [37], the average momentum relaxation time $\tau \sim \ell / v_{F}$ is $\sim 15$ fs in NDG, 34 fs in $\mathrm{AG}$, and $38 \mathrm{fs}$ in TAG. One may try to estimate the conductivity of the graphene in the strong scattering limit [16] by using $\sigma=G_{0}\left(E_{F} \tau / \hbar\right)$, giving $\sigma \sim 6 G_{0}$ for $\mathrm{AG}, \sim 1.4 G_{0}$ for NDG, and $\sim 9 G_{0}$ for TAG. The intensity ratio of $D$ and $D^{\prime}$ bands $I(D) / I\left(D^{\prime}\right)$ has been related to the type of defects [38]. For the AG, $I(D) / I\left(D^{\prime}\right) \sim 7$ implies vacancylike defects, and $I(D) / I\left(D^{\prime}\right) \sim 8.7$ in NDG points to the presence of $s p^{3}$ defects as well [for $s p^{3}$ defects, $I(D) / I\left(D^{\prime}\right) \sim 13$ ] [38].

\section{B. Terahertz conductivity of graphene}

Let the temporal evolution of the transmitted terahertz electric fields through the graphene on the $\alpha$-quartz substrate and through the substrate without graphene be denoted by $T_{\text {sam }}(t)$ and $T_{\text {ref }}(t)$, respectively. The ratio of Fourier transform (FT) of $T_{\text {sam }}(t)$ and $T_{\text {ref }}(t)$ gives amplitude and phase of the spectral transmission function $S(\omega)=\widetilde{T}_{\text {sam }}(\omega) / \widetilde{T}_{\text {ref }}(\omega)$. The complex conductivity spectra can be obtained from the spectral transmission function by using the relation $S(\omega)=\left[n_{s}+1\right] /\left[n_{s}+1+Z_{0} \sigma\right]$ in the limit of thin film approximation $[39,40]$. Here $Z_{0}=377 \Omega$ is the impedance of free space and $n_{s}$ is the refractive index of substrate, taken as 2.2. Figure 2(a) shows the temporal terahertz fields through quartz (black line), AG (red line), and NDG (blue line). From the repeated runs, the error bar at the maximum of the $\mathrm{THz}$ electric field is estimated to be $\sim 8 \%$. The inset shows the signal on an expanded scale near the maximum of the electric field. It can be seen that for NDG, the obtained difference in the peak value of the electric field is $\sim 14 \%$, barely above the uncertainty level of the measurements. Figure 2(b) shows the real part of the conductivity in the spectral range 0.5 to $2.5 \mathrm{THz}$ and the corresponding FTs are shown in the inset. A nearly spectrally flat conductivity suggests large momentum scattering rate for the graphene samples, i.e., $\omega \tau \ll 1[11,19,41]$, as also suggested by the estimates of $\tau$ from the Raman data (see Table I). The average conductivities of AG, NDG, and TAG samples are $(7 \pm 1) G_{0},(1.0 \pm 0.5) G_{0}$, and $(4 \pm 1) G_{0}$, respectively, in close agreement with the estimates obtained from the Raman data. For the NDG sample, the value of the average conductivity is only approximate. The reduction of conductivity of the NDG is due to the shift of the Fermi level towards the Dirac point and a decrease of the momentum relaxation time due to increase in disorder.

TABLE I. Raman characterization.

\begin{tabular}{lcccccc}
\hline \hline Sample & $G\left(\mathrm{~cm}^{-1}\right)$ & $I(2 D) / I(G)$ & $A(D) / A(G)$ & $I(D) / I\left(D^{\prime}\right)$ & Fermi energy, $E_{F}(\mathrm{meV})$ & Crystalline size, $L_{a}(\mathrm{~nm})$ \\
\hline AG & 1583 & 2.4 & 0.6 & 7.0 & -180 & 34 \\
NDG & 1582 & 3.5 & 1.3 & 8.7 & -10 & 15 \\
TAG & 1589 & 1.5 & 0.5 & 5.7 & -200 & 38 \\
\hline \hline
\end{tabular}



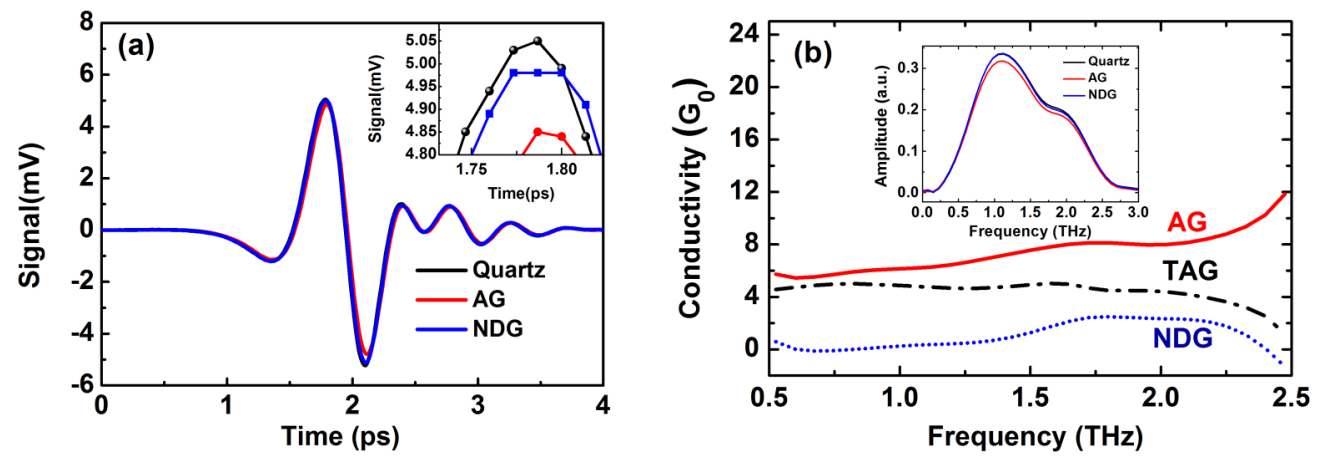

FIG. 2. (Color online) Terahertz conductivity. (a) Evolution of THz electric field as a function of delay time. (b) Conductivity (in unit of $G_{0}$ ) spectra for AG (red solid line), NDG (blue dotted line), and TAG (black dash-dotted line). Inset: FT amplitudes vs frequency.

\section{Optical-pump induced changes in terahertz conductivities}

The differential transmission $\Delta T / T$ is related to the dynamic THz conductivity $\Delta \sigma$ by the relation $\frac{\Delta T}{T} \approx-\frac{Z_{0}}{n_{s}+1} \Delta \sigma$. The results shown in Fig. 3 corresponds to the peak of the $\mathrm{THz}$ electric field using a pump excitation density of $340 \mu \mathrm{J} / \mathrm{cm}^{2}$ per pulse. Taking the absorption at $785 \mathrm{~nm}$ to be $2.3 \%$, photoexcited carrier density is $3 \times 10^{13} / \mathrm{cm}^{2}$ [42]. Most interestingly, $\Delta \sigma$ is negative for the AG, whereas it is positive for the NDG. After annealing the NDG, $\Delta \sigma$ is once again negative for TAG.

To understand our results, we now analyze various contributions to the dynamic conductivity. As mentioned earlier, hot carriers achieve quasiequilibrium Fermi distribution with electron temperature $T_{e}$, thus $\Delta \sigma=\sigma\left(T_{e}\right)-\sigma\left(T_{0}\right)$, where $T_{0}$ is the lattice temperature $(=300 \mathrm{~K})$. The intraband and interband contributions to dynamic conductivity is calculated

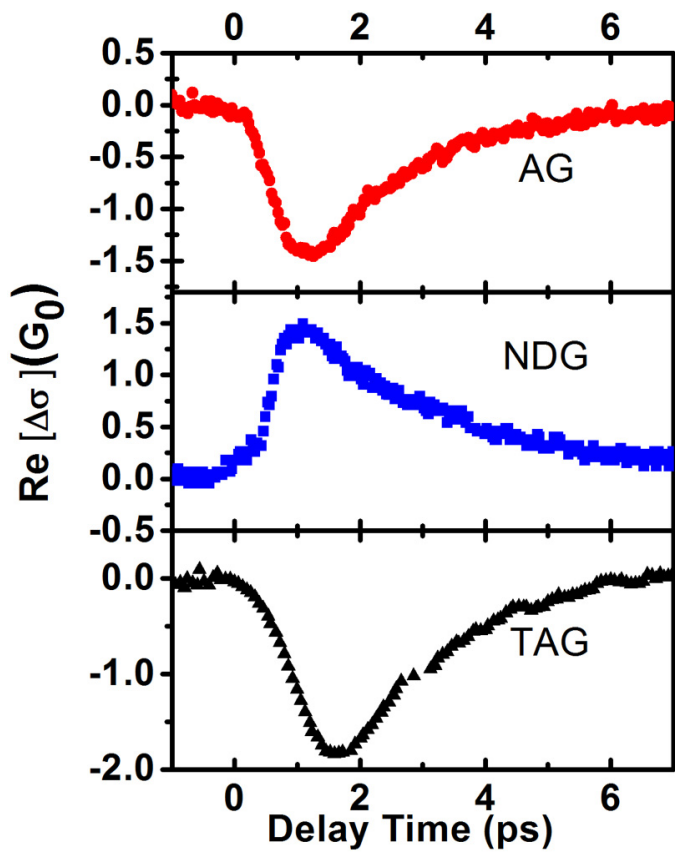

FIG. 3. (Color online) Transient THz photoconductivity. Evolution of the real part of photoinduced $\mathrm{THz}$ conductivity as function of delay time between the $785 \mathrm{~nm}$ pump and the THz probe. using [43]

$$
\begin{gathered}
\sigma_{\text {intra }}(T)=\frac{2 G_{0} \tau k_{B} T}{\hbar\left(\omega^{2} \tau^{2}+1\right)}\left[2 \cosh \left(-\frac{E_{F}}{2 k_{B} T}\right)\right], \\
\sigma_{\text {inter }}(T)=\frac{\pi G_{0}}{8}\left[\tanh \left(\frac{\hbar \omega+2 E_{F}}{4 k_{B} T}\right)+\tanh \left(\frac{\hbar \omega-2 E_{F}}{4 k_{B} T}\right)\right],
\end{gathered}
$$

where $\omega$ is the terahertz-probe frequency. The interband contribution (see Supplemental Material [44], Fig. s1) gives positive and negative $\Delta \sigma$ depending on $E_{F}$ but is $\sim 10^{3}$ times smaller as compared to the intraband contribution for any finite $E_{F}$. Hence the photoinduced terahertz conductivity is dominated by the intraband scattering $[11,19,43]$ and henceforth we will neglect interband contributions. Taking $\tau=34 \mathrm{fs}$, the intraband contribution to the dynamic conductivity at $1 \mathrm{THz}$ is shown in Fig. 4(a) (dotted black line) as a function of Fermi energy at a representative $T_{e}=700 \mathrm{~K}$. In this estimation we have taken into account the change in Fermi energy of graphene as a function of electronic temperature for a fixed carrier concentration. The magnitude of $\Delta \sigma_{\text {intra }}$ as seen in Fig. 4(a) is not sufficient to explain the observed $\Delta \sigma$ in $\mathrm{AG}\left(E_{F} \sim-180 \mathrm{meV}\right)$, which necessarily requires another mechanism.

We consider the recently proposed secondary hot carrier generation (SHCG) [16] in which the photoexcited carriers interact with the intrinsic carriers to excite the later. The photoexcited carriers have two scattering channels; one is the conventional intraband scattering mechanism with momentum relaxation time $\tau$, as discussed earlier, and the other is the Coulomb scattering with momentum relaxation time $\tau_{e}$, which is proportional to carrier energy $\varepsilon$, i.e., $\tau_{e}=b \varepsilon$. Here $b$, the proportionality constant, depends on the ratio of the average interelectron Coulomb interaction energy to the Fermi energy and the density of the secondary hot carriers $\left(n_{i}\right)$ (see Eq. (3.21) of Ref. [45]). The secondary hot carrier generation contribution to the photoinduced conductivity is given by [16]

$$
\begin{gathered}
\Delta \sigma_{\mathrm{el}}=\left.k_{B}^{2}\left(T_{e}^{2}-T_{0}^{2}\right) \frac{\pi^{2}}{6} v\left(E_{F}\right) \frac{\partial^{2} F(\varepsilon)}{\partial \varepsilon^{2}}\right|_{\varepsilon=E_{F}}, \\
F(\varepsilon)=e^{2} v_{F}^{2} \frac{\tau_{e}(\varepsilon)}{1+\omega^{2}\left[\tau_{e}(\varepsilon)\right]^{2}},
\end{gathered}
$$



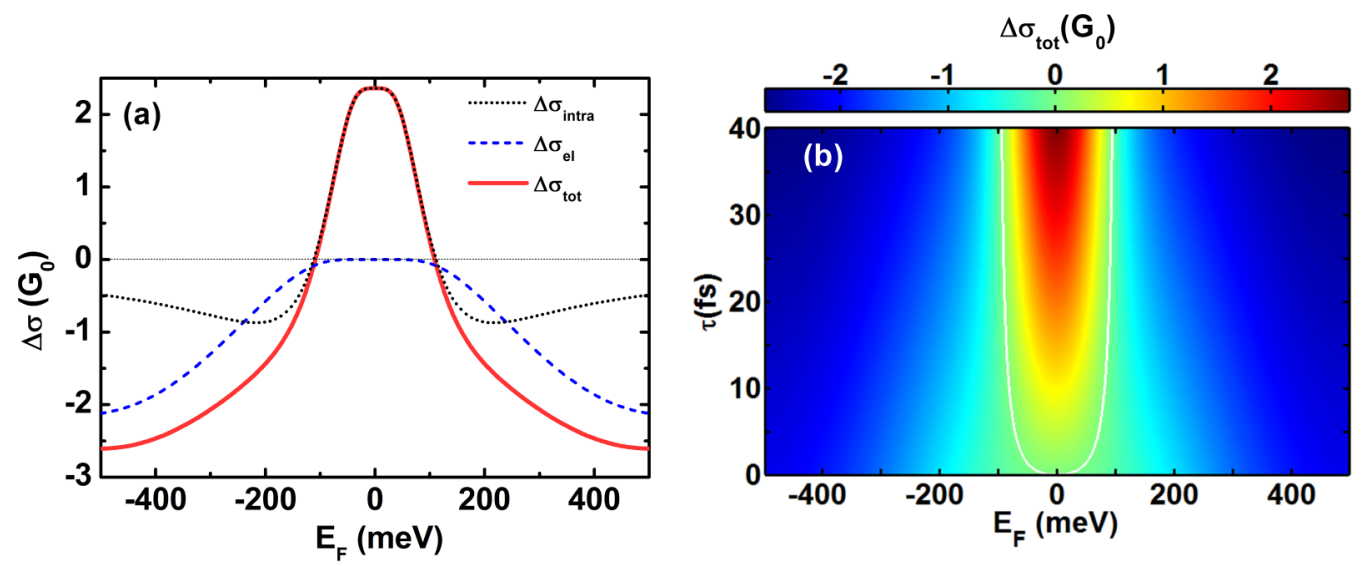

FIG. 4. (Color online) Scattering mechanisms. (a) $\Delta \sigma_{\text {intra }}, \Delta \sigma_{\mathrm{el}}$, and $\Delta \sigma_{\mathrm{tot}}$ are plotted as a function of Fermi energy $E_{F}$ at $T_{e}=700 \mathrm{~K}$, $\tau=34 \mathrm{fs}$, and $b=190 \mathrm{fs} / \mathrm{eV}$ for probe frequency $\omega / 2 \pi=1 \mathrm{THz}$. (b) The contour plot of $\Delta \sigma_{\mathrm{tot}}$ as a function of Fermi energy and momentum relaxation time $\tau$ at $T_{e}=700 \mathrm{~K}$ and $b=190 \mathrm{fs} / \mathrm{eV}$. The white line represents $\Delta \sigma_{\text {tot }}=0$ and indicates the boundary between positive and negative $\Delta \sigma_{\text {tot }}$.

where $v\left(E_{F}\right)$ is density of states of graphene at Fermi energy. As suggested in Ref. [16], we estimate $b=190 \mathrm{fs} / \mathrm{eV}$ after taking $\tau_{e}=34 \mathrm{fs}$ and $E_{F}=-180 \mathrm{meV}$ in calculating $\Delta \sigma_{\mathrm{el}}$. Figure 4(a) (dashed blue line) shows that $\Delta \sigma_{\mathrm{el}}<0$ and the magnitude is comparable to the intraband contribution to the terahertz dynamic conductivity. The total change of terahertz photoconductivity is the sum of intraband and the SHCG contributions: $\Delta \sigma_{\text {tot }}=\Delta \sigma_{\text {intra }}+\Delta \sigma_{\text {inter }}$. Figure 4(b) shows the dynamic terahertz photoconductivity at $1 \mathrm{THz}$ as a function of Fermi energy $E_{F}$ and momentum relaxation time $\tau$ at a representative electron temperature of $700 \mathrm{~K}$. It shows that the magnitude and sign of $\Delta \sigma_{\text {tot }}$ is determined by the value of the Fermi energy and the momentum relaxation time. In AG, $E_{F} \sim-180 \mathrm{meV}$ and $\tau \sim 34 \mathrm{fs}$ gives negative $\Delta \sigma_{\mathrm{tot}}$, whereas a shift of Fermi level towards the Dirac point in NDG results in $\Delta \sigma_{\text {tot }}>0$ due to dominance of the intraband scattering process. Again, in TAG the increased Fermi energy lead to $\Delta \sigma_{\text {tot }}<0$. The results are similar for other representative values of parameter $b(b=120,300 \mathrm{fs} / \mathrm{eV})$ [44].

The dependence of $\mathrm{THz}$ photoconductivity on laser fluence is studied in term of electron temperature. Immediately after pump excitation the sharp change of the $\mathrm{THz}$ conductivity is due to the heating of carriers followed by quasiequilibrium carrier distribution with electron temperature $T_{e}(0)$. Here $T_{e}(0)$ corresponds to maximum of $|\Delta \sigma|$. One can extract $T_{e}(0)$ from $|\Delta \sigma|^{\text {max }}$ by using Eqs. (1) and (3). Figure 5 shows the fluence dependence of increase in electron temperature $\Delta T_{e}(0)$ $\left[=T_{e}(0)-T_{0}\right]$ at the peak of transient conductivity. It is clear that $\Delta T_{e}(0)$ is a sublinear function of fluence and is best described by $\varphi^{1 / 3}$, as shown by solid lines in Fig. 5 . This is expected in graphene due to its linear electronic band dispersion $[13,46]$. However, a few recent works have reported $\varphi^{1 / 2}$ behavior in very highly doped graphene $\left(E_{F}>260 \mathrm{meV}\right)[17,27]$. Since the electron heat capacity of graphene is proportional to the density of state and hence the Fermi energy [27,47], the NDG will have smaller electronic heat capacity than that of $\mathrm{AG}$ and therefore the electron temperature $T_{e}$ in NDG will be more than that in AG for a given pump fluence. This is indeed the case as seen in Fig. 5: $\Delta T_{e}(0)=(380 \pm 34) \mathrm{K}$ in $\mathrm{AG}$ and $\Delta T_{e}(0)=(555 \pm 50) \mathrm{K}$ in NDG at $\varphi=340 \mu \mathrm{J} / \mathrm{cm}^{2}$.

\section{Cooling dynamics}

We now focus on the cooling dynamics of the photoexcited carriers. As mentioned before, due to strong electron phonon coupling the hot carriers can efficiently lose their energy by optical phonon emission within 100-500 fs till their energy comes down to $200 \mathrm{meV}$. Below $200 \mathrm{meV}$, the hot carriers can dissipate energy only by emitting acoustic phonons with energy $k_{B} T_{\mathrm{BG}}$ per scattering event as permitted by conservation of momentum. Here $T_{\mathrm{BG}}$ is a Bloch-Grüneisen temperature given by $k_{B} T_{\mathrm{BG}}=\left(2 v_{s} / v_{F}\right) E_{F}\left(v_{s} \sim 2.1 \times 10^{4} \mathrm{~m} / \mathrm{s}\right.$ is velocity of sound in graphene) which defines a boundary between the low and the high temperature behavior. The cooling of carriers can

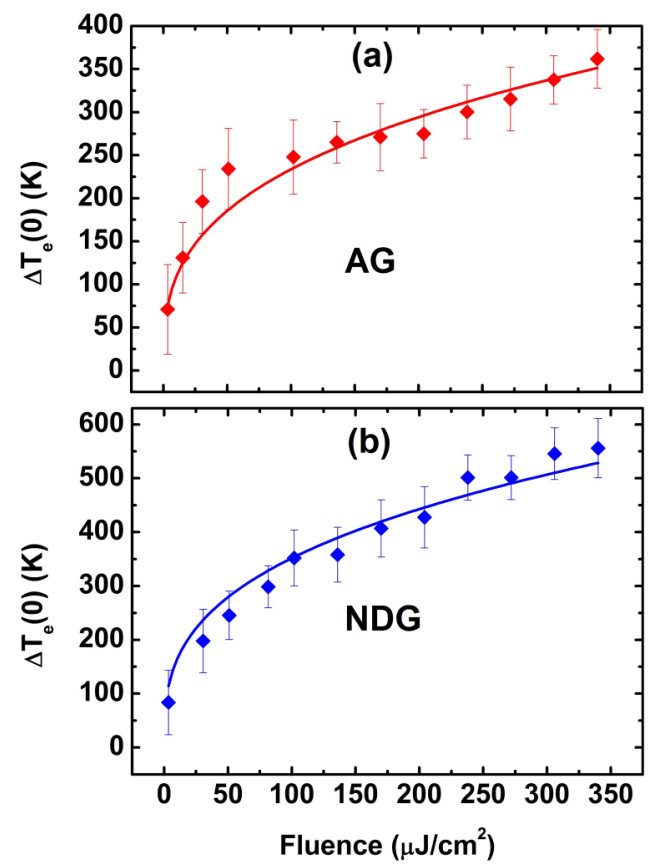

FIG. 5. (Color online) The photoinduced change of electron temperature $\Delta T_{e}(0)$ at the peak of transient conductivity is plotted as a function of fluence for (a) AG and (b) NDG. The solid lines are the fit to $\varphi^{1 / 3}$ 
(a)

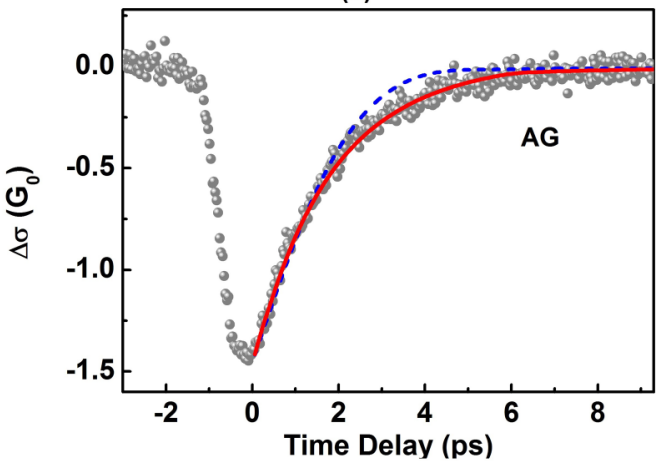

(b)

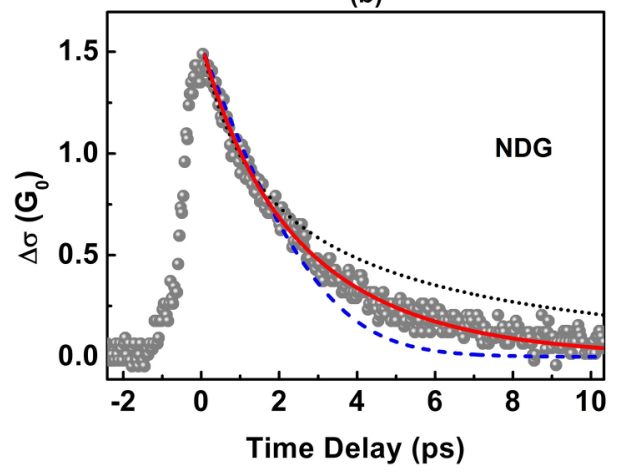

FIG. 6. (Color online) (a) Cooling dynamics of AG. The blue dashed line is fit to normal collision given by Eq. (5) and red solid line is fit to SC given by Eq. (6). (b) Cooling dynamics of NDG. The black dotted curve is fit to normal collision in the nondegenerate limit [Eq. (8)], the blue dashed curve is fit to normal collision in degenerate limit [Eq. (5)], and the red solid curve is fit to SC given by Eq. (6).

last tens of picoseconds for phonon temperature $T_{0}>T_{\mathrm{BG}}$. However, in the presence of disorder an alternate route of energy relaxation is via disorder-mediated emission of high energy $\left(\sim k_{B} T_{e}\right)$ and high momentum $\left(\sim k_{B} T_{e} / \hbar v_{s}\right)$ acoustic phonons, named as supercollision (SC) cooling. With this background we can evaluate different mechanisms to identify the cooling dynamics. The cooling laws are given by $[47,48]$

$$
\begin{aligned}
\frac{\partial T_{e}(t)}{\partial t} & =B \frac{\left(T_{e}-T_{0}\right)}{T_{e}}, \text { for normal collisions } \\
& =A \frac{\left(T_{e}^{3}-T_{0}^{3}\right)}{T_{e}}, \text { for } \mathrm{SC}
\end{aligned}
$$

in the degenerate limit. $B=3 D_{\mathrm{ac}}^{2} E_{F}^{3} / 4 \pi^{2} \rho v_{F}^{4} \hbar^{3} k_{B}$ is the normal cooling rate coefficient and $A=6 \zeta(3) g k_{B} / \pi^{2} k_{F} \ell \hbar$ is the SC cooling rate coefficient. Here $D_{\text {ac }}$ is the deformation potential, $\rho \sim 7.6 \times 10^{-7} \mathrm{~kg} / \mathrm{m}^{2}, \zeta$ is the Riemann zeta function, and $g$ is the electron-phonon coupling strength given by $g=2 D_{\mathrm{ac}}^{2} E_{F} / \rho v_{s}^{2} \pi\left(\hbar v_{F}\right)^{2}$.

The enhancement factor for energy dissipation rate over momentum conserving path ways is expressed as [47]

$$
\frac{H_{\mathrm{SC}}}{H_{0}}=\frac{0.77}{k_{F} \ell} \frac{T_{e}^{2}+T_{e} T_{0}+T_{0}^{2}}{T_{\mathrm{BG}}^{2}} .
$$

Therefore, the enhancement factor depends on temperature, Fermi energy, and disorder. The temperature $T_{\mathrm{BG}}$ is $\sim 87 \mathrm{~K}$ for $\mathrm{AG}$ and $\sim 5 \mathrm{~K}$ for NDG which are less than $T_{0}(=300 \mathrm{~K})$. The enhancement factor from Eq. (7) is $\sim 17380$ for NDG and $\sim 13$ for AG, taking $T_{e}=700 \mathrm{~K}$ and $T_{0}=300 \mathrm{~K}$, clearly showing the dominance of the SC cooling. To further make sure whether SC cooling is the dominant mechanism or not, we fit the dynamic conductivity $\Delta \sigma(t)$ shown in Figs. 6(a) and 6(b),F6 taking into account both normal collisions [Eq. (5)] as well as supercollision [Eq. (6)] together with Eqs. (1), (3), and (4). The blue dashed curve in Fig. 6(a) shows the best fitting to normal collision model with $B=3 \times 10^{14} \mathrm{~K} \mathrm{~s}^{-1}$. This value of $B$ gives very unreasonable high deformation potential $D_{\mathrm{ac}}=260 \mathrm{eV}$. In comparison, the red solid line in Fig. 6(a) shows the best fitting to the SC with $A=5.0 \times$ $10^{8} \mathrm{~K}^{-1} \mathrm{~s}^{-1}$ in AG. This value of $A$ gives $D_{\mathrm{ac}}=17 \mathrm{eV}$, a reasonable value in agreement with reported values [49,50]. Here we have used $k_{F} \ell=\sigma\left(h / 2 e^{2}\right) \sim 6,\left|E_{F}\right|=180 \mathrm{meV}$, and $\tau=34$ fs. This exercise tells us that the SC model explains our data for the AG sample better than the normal collision model.

Coming to NDG [Fig. 6(b)], since $E_{F}$ is close to the Dirac point, we consider the normal collisions in the nondegenerate limit $\left(E_{F} \ll k_{B} T_{e}\right)$ where the cooling law for acoustic phonon emission is given by [48]

$$
\frac{\partial T_{e}(t)}{\partial t}=C T_{e}^{2}\left(T_{e}-T_{0}\right) .
$$

The dotted line in Fig. 6(b) shows the best fitted curve [Eq. (8)] with $C=7.6 \times 10^{5}\left(\mathrm{~K}^{2} \mathrm{~s}\right)^{-1}$, which not only reveals poor fitting but also gives a unphysical value of the deformation potential $D_{\text {ac }}=293 \mathrm{eV}$. Next we test if it is possible to fit the data by normal collision model [Eq. (5)]. Here again, the dashed blue curve in Fig. 6(b) shows poor fitting for normal collision with an unreasonable value of $D_{\text {ac }}=1.95 \times 10^{4} \mathrm{eV}$. The best fitting is obtained by using the SC model [Eq. (6)] with $A=3.6 \times 10^{8} \mathrm{~K}^{-1} \mathrm{~s}^{-1}$, as shown by the solid red line. The extracted $D_{\mathrm{ac}}$ is $\sim 28 \mathrm{eV}$ comparable to the known values [49,50]. Here we have used $\left|E_{F}\right|=10 \mathrm{meV}$ and $k_{F} \ell=\sigma\left(h / 2 e^{2}\right) \sim 1.4$ to extract the deformation potential. Though it is clear that SC cooling dynamics can explain the data very nicely, the confirmation of SC decay law would require lattice temperature dependent cooling measurements that are beyond the scope of this work.

\section{E. Frequency dependence of dynamic conductivity}

We now present the frequency dependence of the dynamic conductivity. After the pump excitation, the photoinduced change in terahertz electric field $\Delta T(t)$ throughout the complete terahertz pulse is measured [44] for a pump fluence of $\sim 340 \mu \mathrm{J} / \mathrm{cm}^{2}$ and Figs. 7(a)-7(c) show the real and imaginary parts of photoinduced conductivity for AG, NDG, and TAG, respectively. In $A G$ the zero crossing of the imaginary part of $\Delta \sigma$ at $1.3 \mathrm{THz}$ clearly suggests a corresponding peak in the real part of $\Delta \sigma$ which can be described by a Lorentzian oscillator. However, the amplitude of the imaginary part is much less than the real part and points to the Drude behavior. Hence the Drude-Lorentz model has been used to describe the 



FIG. 7. (Color online) Photoinduced conductivity $\Delta \sigma(\omega)$ as a function of frequency at 2 ps after photoexcitation for (a) AG, (b) NDG, and (c) TAG. The real parts of the data are shown by opened symbols and imaginary parts are shown by closed symbols. The continuous horizontal lines shows the zero reference lines of $\Delta \sigma(\omega)$. The black and dashed nonlinear black lines are the fit to the Drude-Lorentz model [Eq. (9)].

frequency dependence of $\Delta \sigma(\omega)[20,51,52]$ :

$$
\Delta \sigma(\omega)=\frac{\mathfrak{D} \tau}{1-i \omega \tau}+\frac{i F \omega}{\left(\omega^{2}-\omega_{0}^{2}\right)+i \omega \gamma} .
$$

Here the first term is the Drude part in terms of Drude weight $\mathfrak{D}$ and momentum relaxation time $\tau$. The second term is the Lorentz part with $F$ as the oscillator strength, $\gamma$ as linewidth, and $\omega_{0}$ as the resonant frequency. The fitted curves are shown in Fig. 7(a) for the AG $\left(\mathfrak{D}=1.7 \times 10^{9} \Omega^{-1} \mathrm{~s}^{-1}, \tau=34 \mathrm{fs}\right.$, $\left.F=5.9 \times 10^{8} \Omega^{-1} \mathrm{~s}^{-1}, \gamma=18 \mathrm{THz}, \omega_{0} / 2 \pi=2.3 \mathrm{THz}\right) . \mathrm{In}$ comparison, for the NDG the imaginary part is positive as in the Drude-like response but the positive real part shown

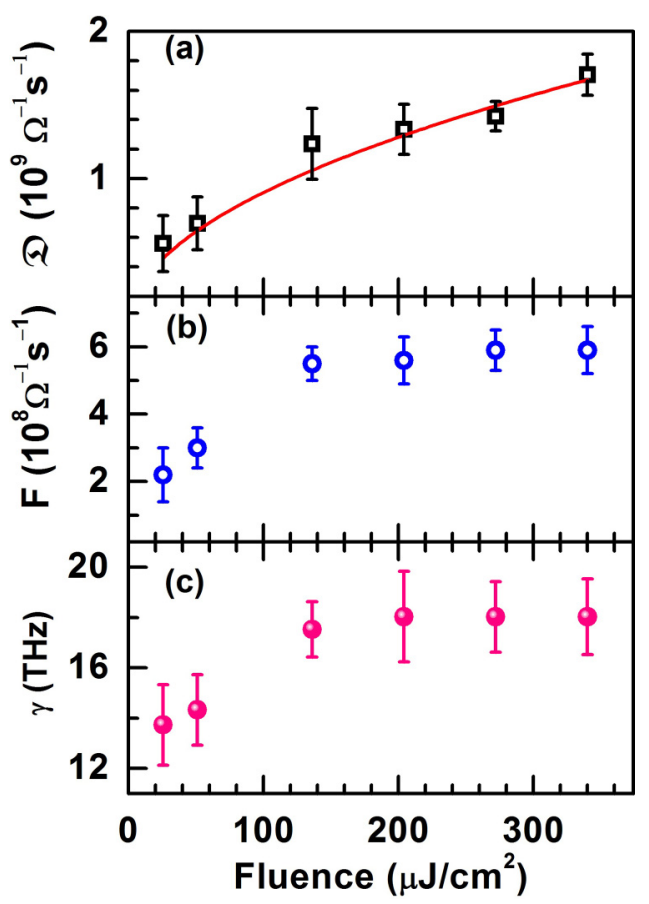

FIG. 8. (Color online) Fitted parameters for AG. (a) The Drude weight $\mathfrak{D}$ varies as $\varphi^{1 / 2}$ (shown by the red solid line). (b) and (c) Oscillator strength $F$ and linewidth $\gamma$ as a function of pump fluence. in Fig. 7(b) cannot be explained by the Drude model. The Drude-Lorentz model [Eq. (9)] is not able to fit the imaginary part of $\Delta \sigma(\omega)$ [Fig. 7(b)], a discrepancy which needs to be understood further. Here we have used $\tau=15$ fs (obtained from Raman scattering) and $\omega_{0} / 2 \pi=2.0 \mathrm{THz}$. The $\Delta \sigma(\omega)$ of TAG is nearly same as that of the AG and fitted with the Drude-Lorentz model with $\mathfrak{D}=1.8 \times 10^{9} \Omega^{-1} \mathrm{~s}^{-1}, \tau=38 \mathrm{fs}$, $F=1.5 \times 10^{9} \Omega^{-1} \mathrm{~s}^{-1}, \gamma=19 \mathrm{THz}$, and $\omega_{0} / 2 \pi=1.7 \mathrm{THz}$, as shown in Fig. 7(c).

The dynamic conductivity $\Delta \sigma(\omega)$ of the AG was measured as a function of pump fluence varying from 25 to $340 \mu \mathrm{J} / \mathrm{cm}^{2}$ [44]. The best fit of the Drude-Lorentz model is obtained by taking $\tau=34 \mathrm{fs}$ and $\omega_{0} / 2 \pi=2.3 \mathrm{THz}$ and varying $\mathfrak{D}, F$, and $\gamma$. The fluence dependence of these parameters are shown in Figs. 8(a)-8(c). It is seen that $\mathfrak{D} \sim \varphi^{1 / 2}$ [see the solid line in Fig. 8(a)], as expected for graphene $\left[\mathfrak{D}=\left(v_{F} e^{2} / \hbar\right) \sqrt{\pi \Delta n}\right]$, where $\Delta n$ is the density of photoinduced carriers participating in intraband scattering.

The physical significance of the resonant frequency $\omega_{0}$ in Lorentz part of $\Delta \sigma$ is not clear. Docherty et al. [20] have considered two possible explanations: excitation of plasmon and a band gap opening. The first possibility in our case is ruled out since $\omega_{0}$ is seen to be fluence independent, similar to Docherty et al. The second explanation in terms of band gap opening of $\sim 9.4 \mathrm{meV}$, equivalent to $\omega_{0} / 2 \pi=2.3 \mathrm{THz}$, seems to be possible, specially in the light of recent work which shows that a band gap can open in graphene due to laser-induced two photon oxidation [53].

\section{CONCLUSION}

In summary, we have presented a quantitative framework of $\mathrm{THz}$ dynamic conductivity in monolayer graphene. We showed that $\Delta \sigma$ is determined by the relative contributions of the secondary hot carrier generation and conventional intraband scattering which, in turn, depend on the position of the Fermi level and momentum relaxation time. In a highly doped sample, the photoexcited hot electrons interact with the intrinsic carriers to generate secondary hot carriers which result in a decrease of the THz conductivity. In NDG, the Fermi 
energy is closer to the Dirac point and the enhanced disorder decreases the momentum relaxation time which makes the intraband scattering as the dominant scattering mechanism resulting in positive $\Delta \sigma$. The cooling dynamics of the hot carriers is well explained in both samples by the disordermediated electron-acoustic phonon interaction, giving the deformation potential comparable to the previous studies. It will be worthwhile to further do OPTP measurements as a function of lattice temperature to further understand the cooling dynamics.

\section{ACKNOWLEDGMENTS}

A.K.S. thanks the Nano Mission Project under the Department of Science and Technology, Government of India for funding. We thank Gyan Prakash for his help in the initial part of the experiments.
[1] F. Bonaccorso, Z. Sun, T. Hasan, and A. C. Ferrari, Nat. Photon. 4, 611 (2010).

[2] K. S. Novoselov, A. K. Geim, S. V. Morozov, D. Jiang, M. I. Katsnelson, I. V. Grigorieva, S. V. Dubonos, and A. A. Firsov, Nature (London) 438, 197 (2005).

[3] S. V. Morozov, K. S. Novoselov, M. I. Katsnelson, F. Schedin, D. C. Elias, J. A. Jaszczak, and A. K. Geim, Phys. Rev. Lett. 100, 016602 (2008)

[4] P. Avouris, Nano Lett. 10, 4285 (2010).

[5] A. K. Geim and K. S. Novoselov, Nat. Mater. 6, 183 (2007).

[6] P. Weis, J. L. Garcia-Pomar, M. Höh, B. Reinhard, A. Brodyanski, and M. Rahm, ACS Nano 6, 9118 (2012).

[7] Q. Bao and K. P. Loh, ACS Nano 6, 3677 (2012).

[8] H. Wang, J. H. Strait, P. A. George, S. Shivaraman, V. B. Shields, M. Chandrashekhar, J. Hwang, F. Rana, M. G. Spencer, C. S. Ruiz-Vargas, and J. Park, Appl. Phys. Lett. 96, 081917 (2010).

[9] H. Choi, F. Borondics, D. A. Siegel, S. Y. Zhou, M. C. Martin, A. Lanzara, and R. A. Kaindl, Appl. Phys. Lett. 94, 172102 (2009).

[10] J. M. Dawlaty, S. Shivaraman, M. Chandrashekhar, F. Rana, and M. G. Spencer, Appl. Phys. Lett. 92, 042116 (2008).

[11] P. A. George, J. Strait, J. Dawlaty, S. Shivaraman, M. Chandrashekhar, F. Rana, and M. G. Spencer, Nano Lett. 8, 4248 (2008).

[12] D. Sun, C. Divin, C. Berger, W. A. de Heer, P. N. First, and T. B. Norris, Phys. Rev. Lett. 104, 136802 (2010).

[13] I. Gierz, J. C. Petersen, M. Mitrano, C. Cacho, I. C. E. Turcu, E. Springate, A. Stöhr, A. Köhler, U. Starke, and A. Cavalleri, Nat. Mater. 12, 1119 (2013).

[14] J. C. W. Song, K. J. Tielrooij, F. H. L. Koppens, and L. S. Levitov, Phys. Rev. B 87, 155429 (2013).

[15] J. C. W. Song, M. S. Rudner, C. M. Marcus, and L. S. Levitov, Nano Lett. 11, 4688 (2011).

[16] K. J. Tielrooij, J. C. W. Song, S. A. Jensen, A. Centeno, A. Pesquera, A. Zurutuza Elorza, M. Bonn, L. S. Levitov, and F. H. L. Koppens, Nat. Phys. 9, 248 (2013).

[17] M. W. Graham, S.-F. Shi, D. C. Ralph, J. Park, and P. L. McEuen, Nat. Phys. 9, 103 (2013).

[18] C. J. Divin, D. Sun, C. Berger, W. de Heer, P. N. First, and T. B. Norris, Conference on Lasers and Electro-Optics/International Quantum Electronics Conference (Optical Society of America, Washington, DC, 2009), p. CMT2.

[19] J. H. Strait, H. Wang, S. Shivaraman, V. Shields, M. Spencer, and F. Rana, Nano Lett. 11, 4902 (2011).

[20] C. J. Docherty, C.-T. Lin, H. J. Joyce, R. J. Nicholas, L. M. Herz, L.-J. Li, and M. B. Johnston, Nat. Commun. 3, 1228 (2012).

[21] G. Jnawali, Y. Rao, H. Yan, and T. F. Heinz, Nano Lett. 13, 524 (2013).
[22] A. J. Frenzel, C. H. Lui, W. Fang, N. L. Nair, P. K. Herring, P. Jarillo-Herrero, J. Kong, and N. Gedik, Appl. Phys. Lett. 102, 113111 (2013).

[23] H. Karasawa, T. Komori, T. Watanabe, A. Satou, H. Fukidome, M. Suemitsu, V. Ryzhii, and T. Otsuji, J. Infrared Millimeter Terahertz Waves 32, 655 (2011).

[24] V. Ryzhii, M. Ryzhii, and T. Otsuji, J. Appl. Phys. 101, 083114 (2007).

[25] A. Satou, V. Ryzhii, Y. Kurita, and T. Otsuji, J. Appl. Phys. 113, 143108 (2013).

[26] T. Otsuji, S. Boubanga-Tombet, A. Satou, M. Suemitsu, and V. Ryzhii, J. Infrared Millimeter Terahertz Waves 33, 825 (2012).

[27] S.-F. Shi, T.-T. Tang, B. Zeng, L. Ju, Q. Zhou, A. Zettl, and F. Wang, Nano Lett. 14, 1578 (2014).

[28] X. Li, W. Cai, J. An, S. Kim, J. Nah, D. Yang, R. Piner, A. Velamakanni, I. Jung, E. Tutuc, S. K. Banerjee, L. Colombo, and R. S. Ruoff, Science 324, 1312 (2009).

[29] R. Lv, Q. Li, A. R. Botello-Mndez, T. Hayashi, B. Wang, A. Berkdemir, Q. Hao, A. L. Elas, R. Cruz-Silva, H. R. Gutirrez, Y. A. Kim, H. Muramatsu, J. Zhu, M. Endo, H. Terrones, J.-C. Charlier, M. Pan, and M. Terrones, Sci. Rep. 2, 586 (2012).

[30] J. W. Suk, A. Kitt, C. W. Magnuson, Y. Hao, S. Ahmed, J. An, A. K. Swan, B. B. Goldberg, and R. S. Ruoff, ACS Nano 5, 6916 (2011).

[31] Y. Wang, Y. Shao, D. W. Matson, J. Li, and Y. Lin, ACS Nano 4, 1790 (2010).

[32] H. Gao, L. Song, W. Guo, L. Huang, D. Yang, F. Wang, Y. Zuo, X. Fan, Z. Liu, W. Gao, R. Vajtai, K. Hackenberg, and P. M. Ajayan, Carbon 50, 4476 (2012).

[33] L. Malard, M. Pimenta, G. Dresselhaus, and M. Dresselhaus, Phys. Rep. 473, 51 (2009).

[34] A. Das, S. Pisana, B. Chakraborty, S. Piscanec, S. K. Saha, U. V. Waghmare, K. S. Novoselov, H. R. Krishnamurthy, A. K. Geim, A. C. Ferrari, and A. K. Sood, Nat. Nano. 3, 210 (2008).

[35] D.-W. Shin, H. M. Lee, S. M. Yu, K.-S. Lim, J. H. Jung, M.-K. Kim, S.-W. Kim, J.-H. Han, R. S. Ruoff, and J.-B. Yoo, ACS Nano 6, 7781 (2012).

[36] L. G. Canado, K. Takai, T. Enoki, M. Endo, Y. A. Kim, H. Mizusaki, A. Jorio, L. N. Coelho, R. Magalhes-Paniago, and M. A. Pimenta, Appl. Phys. Lett. 88, 163106 (2006).

[37] A. H. Castro Neto, F. Guinea, N. M. R. Peres, K. S. Novoselov, and A. K. Geim, Rev. Mod. Phys. 81, 109 (2009).

[38] A. Eckmann, A. Felten, A. Mishchenko, L. Britnell, R. Krupke, K. S. Novoselov, and C. Casiraghi, Nano Lett. 12, 3925 (2012).

[39] J. L. Tomaino, A. D. Jameson, J. W. Kevek, M. J. Paul, A. M. van der Zande, R. A. Barton, P. L. McEuen, E. D. Minot, and Y.-S. Lee, Opt. Express 19, 141 (2011).

[40] M. Tinkham, Phys. Rev. 104, 845 (1956). 
[41] W. Liu, R. Valds Aguilar, Y. Hao, R. S. Ruoff, and N. P. Armitage, J. Appl. Phys. 110, 083510 (2011).

[42] K. F. Mak, M. Y. Sfeir, Y. Wu, C. H. Lui, J. A. Misewich, and T. F. Heinz, Phys. Rev. Lett. 101, 196405 (2008).

[43] S. Winnerl, M. Orlita, P. Plochocka, P. Kossacki, M. Potemski, T. Winzer, E. Malic, A. Knorr, M. Sprinkle, C. Berger, W. A. de Heer, H. Schneider, and M. Helm, Phys. Rev. Lett. 107, 237401 (2011).

[44] See Supplemental Material at http://link.aps.org/supplemental/ 10.1103/PhysRevB.90.165420 for interband conductivity shown in Fig. s1, contour plot of $\Delta \sigma_{\text {tot }}$ shown in Fig. s2, the temporal evolution of photoinduced THz pulse shown in Fig. s3, and spectral dependence of photoinduced complex conductivity of AG at different pump fluences shown in Fig. s4.

[45] S. Das Sarma, S. Adam, E. H. Hwang, and E. Rossi, Rev. Mod. Phys. 83, 407 (2011).

[46] T. Winzer and E. Malić, Phys. Rev. B 85, 241404 (2012).
[47] J. C. W. Song, M. Y. Reizer, and L. S. Levitov, Phys. Rev. Lett. 109, 106602 (2012).

[48] R. Bistritzer and A. H. MacDonald, Phys. Rev. Lett. 102, 206410 (2009).

[49] K. I. Bolotin, K. J. Sikes, J. Hone, H. L. Stormer, and P. Kim, Phys. Rev. Lett. 101, 096802 (2008).

[50] C. R. Dean, A. F. Young, I. Meric, C. Lee, L. Wang, S. Sorgenfrei, K. Watanabe, T. Taniguchi, P. Kim, K. L. Shepard, and J. Hone, Nat. Nano. 5, 722 (2010).

[51] P. Parkinson, J. Lloyd-Hughes, Q. Gao, H. H. Tan, C. Jagadish, M. B. Johnston, and L. M. Herz, Nano Lett. 7, 2162 (2007).

[52] P. Parkinson, H. J. Joyce, Q. Gao, H. H. Tan, X. Zhang, J. Zou, C. Jagadish, L. M. Herz, and M. B. Johnston, Nano Lett. 9, 3349 (2009).

[53] J. Aumanen, A. Johansson, J. Koivistoinen, P. Myllyperkiö, and M. Pettersson, arXiv:1408.0905. 